\title{
Viewpoint \\ Chaperone-mediated destruction of erbB2: relevance to tyrosine kinase inhibitors
}

Julia MW Gee

Tenovus Centre for Cancer Research, Welsh School of Pharmacy, Cardiff University, Wales, UK

Published: 30 July 2002

We are now entering an exciting new era of cancer therapeutics where our goal is to pursue optimal patient outcomes through the use of rationally-designed, target-based anticancer agents. In this light, the four Type 1 erbB plasma membrane-spanning tyrosine kinase receptors (epidermal growth factor receptor [erbB1], erbB2, erbB3 and erbB4), their signal transduction pathways and their inhibition currently comprise the focus of intense research activity. For example, the recent articles by Tan et al. [1], Offterdinger et al. [2], Pusl et al. [3] and Sorkina et al. [4] provide new insights into the effects of erbB2 on the cell cycle, nuclear erbB3, calcium regulation of epidermal growth factormediated transcription, and endocytosis of epidermal growth factor receptor respectively. Among these receptors, the erbB2 oncoprotein (HER2/neu) is of particular interest; successes with this molecule have provided proof of principle that targeting of growth factor receptor signalling is a valid therapeutic option in breast cancer.

erbB2 is the preferred heterodimeric partner for the other erbB family members, potentiating and prolonging their signalling. It is overexpressed in several human cancer types, including 20-30\% of breast tumours. Overexpression, paralleled by gene amplification in $\sim 40 \%$ of cases, triggers kinase activation of the receptor and, as a consequence, deregulated signal transduction. Increased erbB2 activation has been reported to potentiate tumour cell motility, protease secretion and invasion, and to modulate cell cycle checkpoint function, DNA repair and cell survival. Not surprisingly, therefore, erbB2 overexpression in breast cancer is associated with aggressive disease biology, resistance to tamoxifen treatment and reduced patient survival. As such, there has been considerable interest in inhibition of this receptor. To date, erbB2 directed therapies have largely focused around the use of immunoreagents, because erbB2 is accessible on the cancer cell surface. Such reagents have obvious growth-inhibitory activity and can also potentially provide effective vehicles for toxin delivery to cells. Of particular note is the recombinant humanised erbB2 antibody Herceptin ${ }^{\mathrm{TM}}$ (trastuzumab) which has anti-tumour activity as a single agent in patients with erbB2
Breast Cancer Res 2002, 4:205-206

(C) 2002 BioMed Central Ltd

(Print ISSN 1465-5411; Online ISSN 1465-542X) overexpressing advanced disease, and shows additive benefits with chemotherapeutic strategies in improving objective response and overall survival in metastatic breast cancer. The growth-inhibitory mechanism of the erbB2 antibody Herceptin is believed to be quite complex. However, a key consequence of antibody binding to the extracellular domain of erbB2 appears to be enhanced removal of the receptor from the cell surface, with subsequent inhibition of receptor signalling. Further improvements in the design of erbB2-specific immunoreagents are ongoing (for example, see article by De Lorenzo et al. [5] describing isolation and demonstration of growth inhibitory capabilities of a novel human mini erbB2 antibody).

Additional therapeutic approaches with considerable potential have also emerged in the quest to effectively target erbB2. Firstly, clinical trials are underway to examine the safety and efficacy of antagonists of heat shock protein 90 (Hsp90), a chaperone protein that maintains client proteins (in particular erbB2) in a signallingcompetent form. Hsp90 inhibitors, including the ansamycin antibiotic geldanamycin, appear able to reduce erbB2 levels, thereby exerting marked growth-inhibitory effects in erbB2 overexpressing cancer cells. Geldanamycin has been shown to avidly bind and inactivate the ATP binding pocket in Hsp90 protein, a key regulatory region for chaperone function. This inhibition of chaperone function triggers erbB2 dissociation from Hsp90. As a consequence of dissociation, there is ubiquitylation and proteasomal destruction of the erbB2 protein resulting in reduced levels in the cell.

Secondly, small molecule receptor tyrosine kinase inhibitors (TKIs) specifically targeting erbB receptors are showing much promise and their clinical testing is ongoing. Simplistically, the growth inhibitory activity of TKls resides in their ability to bind to the kinase domains of erbB receptors, blocking ATP binding to abrogate erbB-mediated signalling. The new findings from Citri et al. [6] now reveal a further fascinating dimension to the mode of action of TKls that may provide as yet unexplored thera- 
peutic avenues. TKls also appear able to partially deplete erbB2 in overexpressing tumour cells, with the irreversible pan-erbB tyrosine kinase inactivator $\mathrm{Cl}-1033$ proving particularly potent in this regard. This down-regulation parallels drug-induced tumour cell growth arrest. TKI-induced loss of erbB2 again involves targeting of receptor interactions with molecular chaperones, thereby resulting in erbB2 destruction. However, Citri et al. [6] propose a model where the TKI-induced degradation mechanism of erbB2 differs subtly from that of geldanamycin. TKI binds to and perturbs the ATP-binding pocket of erbB2 thereby destabilising the complex with Hsp90, rather than acting to block ATP binding to the Hsp90 protein. Interestingly, the end-result of this TKI-induced distortion of the erbB2 kinase conformation is equivalent to that of geldanamycin, in that there is recruitment of an alternative protein Hsp70, erbB2 poly-ubiquitylation, accelerated endocytosis and intracellular receptor destruction. An additive effect with regards to erbB2 degradation and anti-tumour activity was observed in geldanamycin/ $\mathrm{Cl}-1033$ combination experiments, which supports the working hypothesis that these inhibitory agents act on different components of the chaperone-receptor complex in their direction of erbB2 to a common degradation pathway.

Increasing knowledge of such degradation mechanisms may enable improvement of tyrosine kinase inhibitors or the design of "smart" treatment combinations, as well as novel drug development to efficiently enhance erbB2 downregulation in cells. Moreover, it is envisaged that this concept could have broader significant therapeutic implications in breast cancer because it may prove applicable to other erbB tyrosine kinase receptors and additional pivotal chaperone-controlled oncoproteins, for example insulin-like growth factor-1 receptor, Raf-1, mutant p53, and steroid hormone receptors.

\section{Articles selected from Faculty of $\mathbf{1 0 0 0}$}

1. Tan $M$, Jing $T$, Lan $K H$, Neal $C L$, Li $P$, Lee $S$, Fang $D$, Nagata $Y$, Liu J, Arlinghaus R, Hung MC, Yu D: Phosphorylation on tyrosine-15 of p34(Cdc2) by ErbB2 inhibits p34(Cdc2) activation and is involved in resistance to taxol-induced apoptosis. Mol Cell 2002, 9:993-1004

For the Faculty of 1000 evaluation of this article please see http://breast-cancer-research.com/reports/bcr8_02.asp\#tan

2. Offterdinger M, Schöfer C, Weipoltshammer K, Grunt TW: cerbB-3: a nuclear protein in mammary epithelial cells. J Cell Biol 2002, 157:929-939.

For the Faculty of 1000 evaluation of this article please see http://breast-cancer-research.com/reports/bcr8_02.asp\#offterdinger

3. Pusl T, Wu JJ, Zimmerman TL, Zhang L, Ehrlich BE, Berchtold MW, Hoek JB, Karpen SJ, Nathanson MH, Bennett AM: Epidermal growth factor-mediated activation of the ETS-domain transcription factor Elk-1 requires nuclear calcium. J Biol Chem 2002; 277:27517-27527.

For the Faculty of 1000 evaluation of this article please see http://breast-cancer-research.com/reports/bcr8 02.asp\#pus|

4. Sorkina T, Huang F, Beguinot L, Sorkin A: Effect of tyrosine kinase inhibitors on clathrin coated pit recruitment and internalization of epidermal growth factor receptor. J Biol Chem 2002; 277:27433-27441.
5. De Lorenzo C, Palmer DB, Piccoli R, Ritter MA, D'Alessio G: A new human antitumor immunoreagent specific for ErbB2. Clin Cancer Res 2002, 8:1710-1719.

For the Faculty of 1000 evaluation of this article please see http://breast-cancer-research.com/reports/bcr8_02.asp\#lorenzo

6. Citri A, Alroy I, Lavi S, Rubin C, Xu W, Grammatikakis N, Patterson C, Neckers L, Fry DW, Yarden Y: Drug-induced ubiquitylation and degradation of ErbB receptor tyrosine kinases: implications for cancer therapy. EMBO J 2002, 21:2407-2417. For the Faculty of 1000 evaluation of this article please see http://breast-cancer-research.com/reports/bcr8_02.asp\#citri 